\title{
Alternative method of sampling placentas for the assessment of uteroplacental vasculature
}

\author{
TY Khong, H M Chambers
}

\begin{abstract}
A new method for the taking and embedding of placental blocks to obtain maximum information regarding the uteroplacental vasculature was developed. This method involves taking en face blocks of the basal plate of the placenta. Results show that normal and abnormal maternal vessels are clearly delineated.

In addition to conventional full thickness blocks, which provide information about the chorionic plate such as inflammation and meconium pigment uptake, and about the parenchyma in the other zones remote from the basal plate of the placenta, it is recommended that en face blocks be taken for histological assessment of the placenta.
\end{abstract}

(F Clin Pathol 1992;45:925-927)

During normal pregnancy, extravillous trophoblast invading maternal decidua and myometrium transform the normally narrow calibre spiral arteries into distended uteroplacental arteries. These changes constitute the physiological vascular changes of pregnancy and effect an increased blood flow to the placenta and fetus. ${ }^{12}$ It is now evident that an assessment of the maternal uteroplacental vasculature is an important and necessary component of the examination of the placenta in numerous pregnancy disorders, including pre-eclampsia, intrauterine fetal growth retardation, miscarriage and maternal connective tissue disease. ${ }^{1-6}$ Definitive evidence of abnormal placentation includes absence of physiological vascular changes, acute atherosis, and, in the third trimester, intraluminal endovascular trophoblast.

Histological assessment of the maternal blood supply to the placenta can be made either by a study of placental bed biopsy specimens or of the basal plate of the placenta. ${ }^{3}$ The former is usually attainable only at caesarean section or else not available in every obstetric centre. Thus it is necessary to maximise the information available from the examination of the placental basal plate. The current recommendation for histological examination is that sampling consist of full thickness blocks of the placenta extending from the fetal to the maternal surface, including both amnion and decidua. $^{78}$ However, these sections do not always contain maternal vessels for assessment. It occurred to one of us (TYK) that an alternative method for examining these utero- placental arteries would be to take en face blocks of the placental basal plate which would comprise a larger surface area and, accordingly, have a greater chance of including a spiral artery for assessment. This paper reports the preliminary results of this alternative method of taking and embedding placental blocks and a comparative quantitative assessment between conventional and alternative block taking.

\section{Methods}

All placentas submitted to the Department of Pathology, Queen Victoria Hospital in a 14 week period (April to July 1991) were entered into the study. Conventional blocks were taken to include the full thickness of the parenchyma from the amniotic surface through to the basal plate. A random en face block of the basal plate was also taken from each placenta (or corresponding half of a fused twin placenta) by shaving the basal plate and embedding the block on its face. All sections were examined and the number of uteroplacental arteries in the conventional blocks and alternative en face blocks were compared. Specifically, the presence or absence of physiological changes ${ }^{23}$ and the presence of the vasculopathy, acute atherosis, $^{9-11}$ in these uteroplacental arteries were documented in each case.

\section{Results}

A total of 136 placentas were submitted for histopathological examination. There were 33 twin and two triplet placentas. In three twin placentas an en face block was taken from only one half of the placenta; therefore, 170 sets of placentas were available for comparison. Fifty five pregnancies were complicated by conditions thought to be associated with maternal vascular abnormalities (intrauterine growth retardation $(n=20)$, pre-eclampsia $(n=19)$, intrauterine growth retardation and preeclampsia $(n=4)$, systemic lupus erythematosus or mixed connective tissue disease $(n=3)$ and placental abruption $(n=9)$.

The other 81 placentas were sent for various reasons: preterm delivery $(n=41)$, ascertainment of zygosity in multiple pregnancy $(n=20)$, unusual gross lesions in the placenta $(\mathrm{n}=3)$, fetal distress or fetal anomaly $(n=11)$ and other maternal or obstetrical reasons, such as hepatitis, diabetes mellitus, placenta praevia and polyhydramnios $(n=6)$.

When blocks were taken en face to the basal 


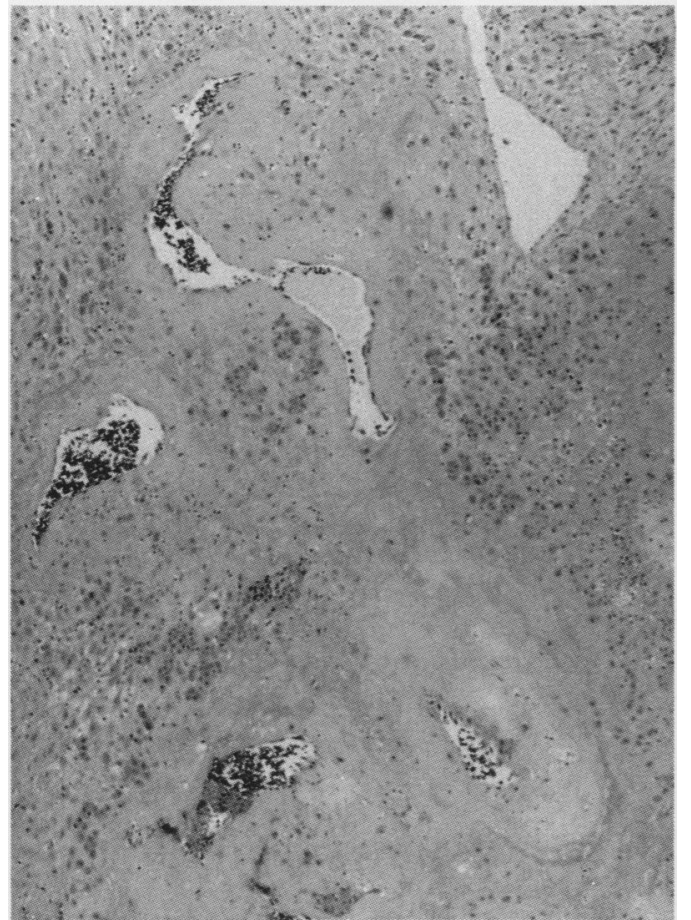

Figure 1 Section showing a leash of uteroplacental arteries in an en face block of placental basal plate. Note the distended lumina.

plate, occasionally the morphological picture was that of islands of villi with basal plate or superficial decidua very much like the hillocks and valleys seen in a topographical map. Uteroplacental arteries were defined as those arteries with fibrinoid matrix in the walls in which were embedded endovascular trophoblast (fig 1). Absence of physiological vascular changes could be seen in the form of unconverted spiral arteries that retained their mus-

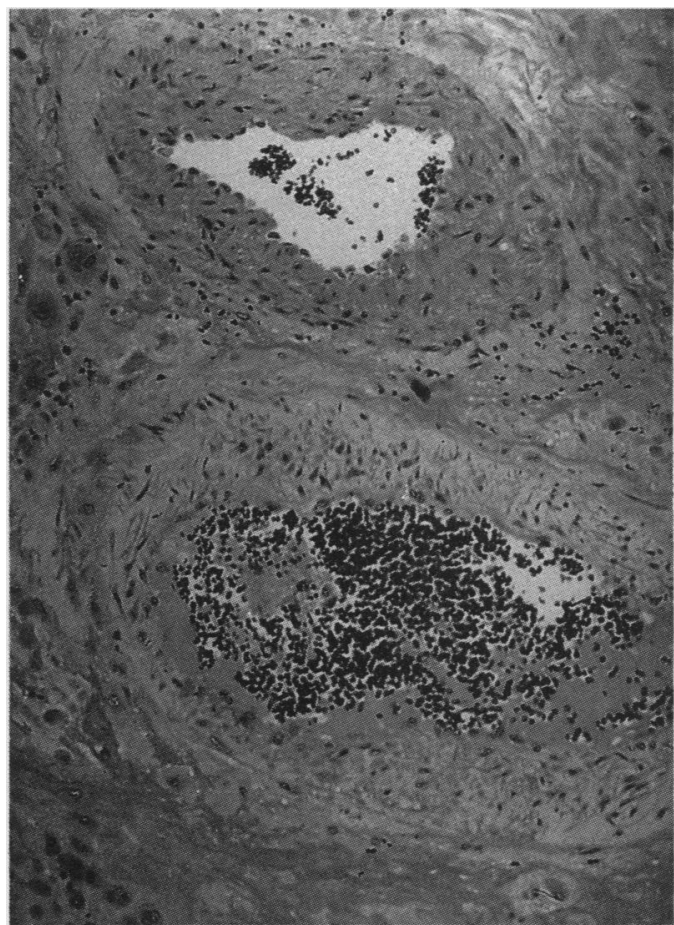

Figure 2 En face basal plate block: spiral arteries show no evidence of physiological changes with retention of muscular and elastic tissue within their walls and narrow calibre lumen.

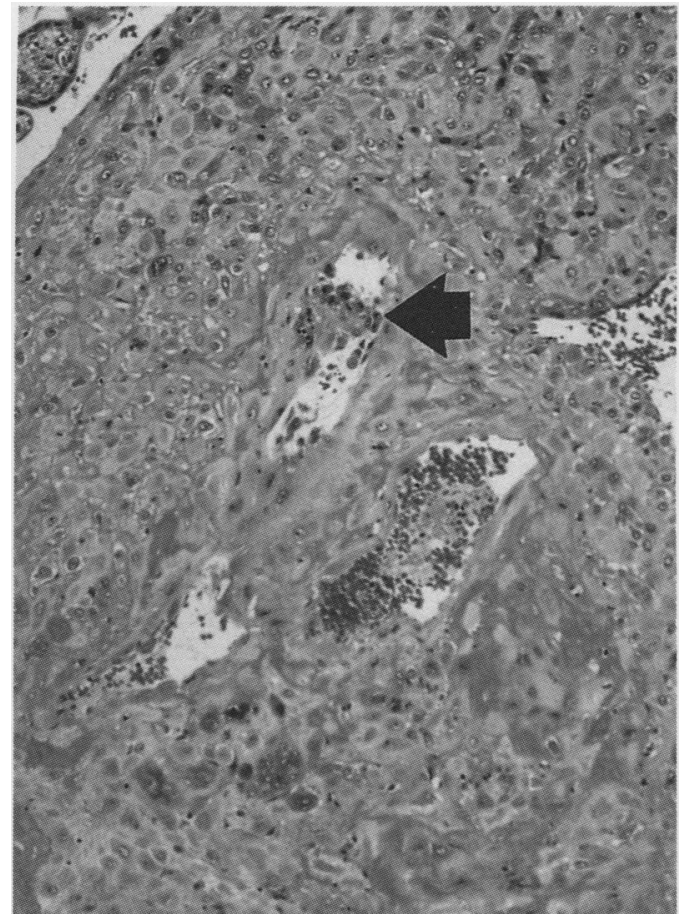

Figure 3 En face basal plate block: intraluminal endovascular trophoblast (arrow) in a pregnancy complicated by intrauterine growth retardation.

cular and elastic tissue within the walls (fig 2); this was observed only in pregnancies complicated by pre-eclampsia, or intrauterine growth retardation, or both. A further abnormal finding was the presence of endovascular trophoblast either free in the lumen or attached to the intima in the abnormal pregnancies (fig 3). Acute atherosis could be identified in the en face blocks (fig 4).

Uteroplacental arteries-spiral arteries

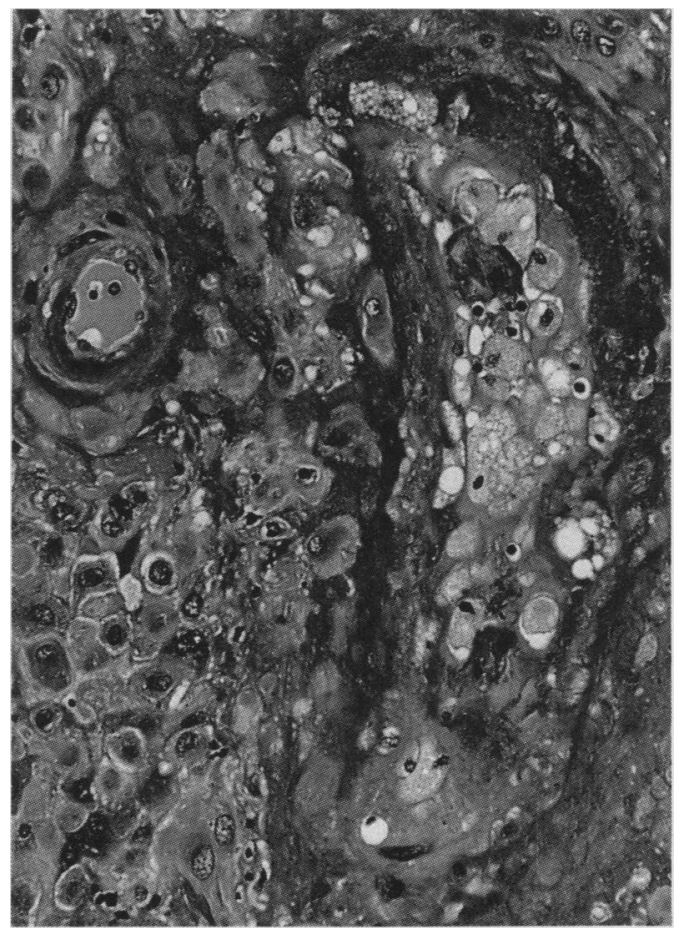

Figure 4 En face basal plate block: acute atherosis in a pregnancy complicated by fulminating pre-eclampsia and intrauterine growth retardation. 
showing physiological changes, were seen in 91 en face blocks and in $\mathbf{3 8}$ full thickness blocks, while spiral arteries devoid of physiological changes were seen in two en face and two full thickness blocks of the same two cases. Thus more vessels could be seen in the en face basal plate blocks more often than could be obtained in the full thickness blocks (93 or $54.7 \% v 40$ or $23.5 \%$ ). For the detection of uteroplacental arteries en face blocks were superior to full thickness blocks in 80 cases (in 69 corresponding full thickness blocks no spiral arteries were detected), inferior in 16 cases (no arteries were detected in 16 en face blocks) and no different in 72 cases (no arteries were detected in 61 en face or full thickness blocks). Features of abnormal placentation were seen more often also in en face blocks: intraluminal endovascular was seen in five en face but not in any full thickness blocks while acute atherosis was seen in one case in only one en face block. Villitis of unknown aetiology was clearly seen in the en face blocks in seven cases.

\section{Discussion}

More maternal spiral arteries could be identified by embedding an en face block of the basal plate than by conventional full thickness blocks. This confirms previous observations ${ }^{10}$ that the basal plates from full thickness blocks often do not include maternal vessels. Utilising full thickness blocks taken at every $20 \mathrm{~mm}$ along the maximum diameter of the placenta and thus yielding six to eight blocks per placenta, Khong found spiral arteries in only $73 \%$ of placentas, ${ }^{10}$ an expensively low yield, considering the number of blocks. The pick-up rate of $54.7 \%$ from one en face block in the present study compares very favourably.

The finding of uteroplacental arteries showing physiological changes in the basal plate is not a reliable indicator of normality because deeper decidual and myometrial segments may not show such changes. ${ }^{3}$ The more diagnostically useful feature is the finding of spiral arteries without physiological changes and, in this respect, these were seen equally frequently in en face and full thickness blocks. It could therefore be argued that en face blocks offer no advantage over full thickness blocks. However, we used only one en face block in this evaluation and we could detect more maternal arteries more frequently in en face than full thickness blocks, allowing a theoretically increased likelihood of detecting unconverted arteries. Furthermore, en face blocks proved superior in detecting other parameters of abnormal placentation, such as acute atherosis $^{911}$ or intraluminal endovascular trophoblasts. $^{3}$

En face blocks also proved useful in detecting villitis of unknown aetiology as these lesions are often distributed among anchoring villi in some cases. ${ }^{12}$ No valid comment can be made regarding its comparison with full thickness blocks because these lesions are often focal.

A potential pitfall of this method of sampling for assessing uteroplacental arteries was the possible confusion between the unconverted spiral arteries and the thicker muscular arteries of the mainstem villi. However, the presence of an investing layer of syncytial trophoblast in these mainstem villi, surrounding fetal mesenchyme and the absence of surrounding decidua or trophoblast easily distinguished fetal and maternal arteries.

Preliminary results of this work were presented to the 36th Annual Scientific meeting of The Royal College of Pathologists of Australasia, September 1991.

We gratefully acknowledge the technical assistance of FM Hill, AR Foreman, C Mott and M Tamblyn.

1 Khong TY. The Robertson-Brosens-Dixon hypothesis: evidence for the role of haemochorial placentation in pregnancy success. $\mathrm{Br} \mathscr{J}$ Obstet Gynaecol 1991;98: pregnancy.

2 Robertson WB, Khong TY, Brosens I, De Wolf F, Sheppard BL, Bonnar J. The placental bed biopsy. A review from three European centers. Am $\mathcal{F}$ Obstet Gynecol 1986; 155:401-12.

3 Khong TY, De Wolf F, Robertson WB, Brosens I. Inadequate maternal vascular response to placentation in pregnancies complicated by pre-eclampsia and by smallfor-gestational age infants. $\mathrm{Br} \mathcal{F}$ Obstet Gynaecol 1986; 93:1049-59.

4 Khong TY, Liddell HS, Robertson WB. Defective haemochorial placentation as a cause of miscarriage: a
preliminary study. $B r \mathcal{F}$ Obstet Gynaecol 1987;94:649-55.

5 Rushton DI. The placenta as a reflection of maternal disease. In: Perrin EVDK, ed. Pathology of the Placenta. disease. In: Perrin EVDK, ed. Pathology of the

6 Wells M, Bulmer JN. The human placental bed: histology, immunohistology and pathology. Histopathology 1988; 13:483-98.

7 Fox H. Pathology of the placenta. London: W B Saunders, 1978.

8 Driscoll SG, Langston C. College of American Pathologists Conference XIX on the examination of the placenta: report of the working group on methods for placental examination. Arch Pathol Lab Med 1991;115:704-8.

9 Khong TY. Acute atherosis in pregnancies complicated by hypertension, small-for-gestational age infants and diabehypertension, small-for-gestational age infants and

10 Khong TY. Morphological and immunohistochemical studies in human normal and abnormal placentation. (MD Thesis) University of Sheffield, 1987:39-61.

11 Labarrere CA. Acute atherosis: a histopathological hall mark of immune aggression? Placenta 1988;9:95-108.

12 Labarrere CA. Allogeneic recognition and rejection reactions in the placenta. Am $\mathcal{F}$ Reproduct Immunol 1989; 21:94-9. 